\title{
Endoglin (CD105) and SMAD4 regulate spheroid formation and the suppression of the invasive ability of human pancreatic cancer cells
}

\author{
ERI KOKAJI ${ }^{1,2}$, AKIKO SHIMOMURA ${ }^{1}$, TAKASHI MINAMISAKA ${ }^{1,2}$, TAKAHIKO NAKAJIMA ${ }^{1}$, \\ SHIGEHARU MIWA ${ }^{1}$, HIDEKI HATTA ${ }^{1}$, TAKESHI NISHIDA ${ }^{1}$, CHIEKO KIYA $^{2}$ and JOHJI IMURA ${ }^{1,2}$ \\ ${ }^{1}$ Department of Diagnostic Pathology, Graduate School of Medicine and Pharmaceutical Sciences, University of Toyama; \\ ${ }^{2}$ Department of Diagnostic Pathology, Toyama University Hospital, Toyama 930-0194, Japan
}

Received November 8, 2017; Accepted January 25, 2018

DOI: 10.3892/ijo.2018.4262

\begin{abstract}
In this study, we investigated the ability of pancreatic cancer cell lines to form spheroids with the aim of identifying factors involved in cell invasiveness, a property that leads to a poor prognosis in pancreatic cancer. For this purpose, 8 cell lines derived from human pancreatic cancer tissues were cultured in non-adherent culture conditions to form spheroids, as well as normal monolayers. The morphology of the cells was observed and spheroid diameters measured. mRNA expression was compared between cells cultured under both culture conditions. The gene knockdown of endoglin (ENG) and SMAD4, components of the transforming growth factor- $\beta$ (TGF- $\beta$ ) signaling system, using siRNAs was conducted in spheroids in order to identify affected protein signaling factors, determine the morphological changes occurring over time and to measure the invasive capacity of the cells constituting spheroids. The cell lines exhibited differences in their spheroid-forming abilities. The expression of SMAD4 and ENG concomitantly increased in the cells that formed spheroids. SMAD4 was transported into the nucleus when spheroids were formed. The expression of ENG was decreased in the cells in which SMAD4 was knocked down; by contrast, the expression of BMP and activin membrane-bound inhibitor (BAMBI) and noggin (NOG), further components of the TGF- $\beta$ signaling system, increased. In the cells in which ENG was knocked down, the decreased mRNA expression of TGF- $\beta$ receptor type 2 (TGFBR2) and SMAD9 was observed, as well as a change in the expression of pSMAD1/5/9, and a tendency of spheroids to decrease in size. Spheroids cultured
\end{abstract}

Correspondence to: Dr Johji Imura, Department of Diagnostic Pathology, Graduate School of Medicine and Pharmaceutical Sciences, University of Toyama, 2630 Sugitani, Toyama 930-0194, Japan E-mail:imura@med.u-toyama.ac.jp

Key words: endoglin (CD105), SMAD family member 4, spheroid, invasion, pancreatic cancer on Matrigel exhibited a tendency towards a reduction in size over time, as well as a tendency to invade into the Matrigel. In particular, the cells in which ENG was knocked down exhibited spheroids which were reduced in size, and also exhibited an increase in invasiveness, and a decrease in adhesiveness. Thus, our data indicate that in pancreatic cancer cells, the expression of ENG may be controlled by a pathway mediated by SMAD4. In addition, ENG was found to be related to the spheroidforming ability of cells and to be involved in the invasive capacity of pancreatic cancer cells.

\section{Introduction}

Worldwide, the mortality rate due to pancreatic cancer is higher than that of other organ malignancies, with pancreatic cancer being one of the tumors associated with the poorest prognosis (1). The reasons for this include the difficulty in the early detection of pancreatic cancer due to the pancreas being an underlying organ and due to the fact that the disease is already at an advanced stage by the time the symptoms manifest themselves. Furthermore, pancreatic cancer easily invades into adjacent organs, with the frequency of metastasis to other organs also being high (2). As mentioned above, a number of observed features define the prognosis of pancreatic cancer; however, the biological characteristics of this type of cancer may also strongly contribute to the prognosis. More than $90 \%$ of pancreatic cancer cases are pancreatic ductal adenocarcinoma (PDAC) that develops from the pancreatic ductal epithelium (3); however, the development and progression of PDAC remain unclear. In particular, the progression of PDAC is associated with characteristics that are not found in malignant tumors of other organs. For example, pancreatic cancer tissues become highly fibrotic, allowing tumor cells to easily migrate and invade into such an abundant tumor stroma. Similar to tumors affecting other organs, pancreatic tumor cells can migrate and invade as single cells; however, they are also characterized by the ability to invade, while maintaining their binding ability between individual tumor cells, and also their glandular structure (4). The mechanisms associated with an invasive phenomenon whereby cells invade into the solid stroma as a group, rare in malignant tumors affecting other 
organs, are unexplained, and many aspects have yet to be clarified.

Genetic abnormalities define the biological properties of tumor cells (5). Previous studies have demonstrated this to also be true in pancreatic cancer. Particular and specific genetic abnormalities have been found more commonly in pancreatic cancer as compared with malignant tumors affecting other organs (6). A KRAS genetic abnormality is representative of such abnormalities, and point mutations of KRAS have been found in $\sim 80 \%$ of pancreatic cancers (7). In addition, genetic abnormalities in CDKN2A (8), TP53 (9), SMAD4 (10) and in other genes occur more frequently in the pancreas and are more specific than in other organs. Of these genes, SMAD4 is inactivated in about a half of all pancreatic cancers although this is generically known as a tumor suppressor gene (11). As with $K R A S$, the frequency of SMAD4 inactivation is low in malignant tumors affecting other organs. Furthermore, the inactivation of SMAD4 is related to an exacerbation of the prognosis of pancreatic cancer (12). SMAD4 travels into the nucleus after forming a complex with R-SMAD, which is phosphorylated by a ligand, such as transforming growth factor- $\beta$ (TGF- $\beta$ ) and bone morphogenetic protein (BMP) acting on the TGF- $\beta$ receptor, and, as a transcription factor, regulates the expression of a number of genes (13). In comparison, endoglin (ENG, also known as CD105) is a transmembrane glycoprotein of $\sim 180 \mathrm{kDa}$ in size, known as a TGF- $\beta$ accessory receptor (14). ENG is expressed in vascular endothelial cells, particularly at high levels in growing vascular endothelial cells, and is involved in angiogenesis (14). Few studies exist on the expression of ENG in tumor cells themselves; however, its expression in gastrointestinal stromal tumors (15), as well as breast (16), ovarian (17) and pancreatic $(18,19)$ cancers, has been confirmed.

This study aimed to clarify the mechanisms involved in pancreatic cancer cell invasion, while maintaining duct formation, a peculiar biological characteristic of pancreatic cancer in vitro. More specifically, we used cell lines derived from human pancreatic cancers in order to clarify the association of ENG, found after searching for factors related to spheroid formation in such cells, with epithelial-mesenchymal transition (EMT), as well as to elucidate the mechanisms responsible for the migration and invasion of pancreatic cancer cells.

\section{Materials and methods}

Cell lines and cell culture. The human pancreatic cancer cell lines, AsPC-1, BxPC-3, KP-2, KP-3, MIA-PaCa, Panc-1, SUIT-2 and TCC-PAN2, were used. The AsPC-1, BxPC-3, MIA-PaCa and Panc-1 cell lines were purchased from the American Type Culture Collection (ATCC, Manassas, VA, USA), the KP-2 and KP-3 cell lines were from the Kyushu Cancer Center (Fukuoka, Japan), and the SUIT-2 and TCC-PAN2 cell lines were from the Japanese Collection of Research Bioresources Cell Bank (Osaka, Japan). Spheroids were formed using EZSPHERE ${ }^{\circledR}$ (spheroid forming culture ware, AGC Techno Glass, Shizuoka, Japan) according to a three-dimensional (3D) culture method. As culture ware with a concave bottom surface and non-adherent treatment was used in this method, the cells adhered to each other, but not with the bottom surface. In addition, the cells did not extend themselves, but formed spheroids as a result. A monolayer (2D) culture method using Petri dishes with adherent treatment was used for the control group. The cells were cultured in RPMI-1640 medium containing 10\% FBS (both from Gibco/Thermo Fisher Scientific, Waltham, MA, USA), antibacterial agents (penicillin and streptomycin) and anti-mycotic agents (amphotericin B) under $5 \% \mathrm{CO}_{2}$ at $37^{\circ} \mathrm{C}$. The cells were grown until spheroids formed for the $3 \mathrm{D}$ cultures, and until subconfluent conditions for the 2D cultures. The spheroids obtained by $3 \mathrm{D}$ culture were collected with the culture medium without losing cell junctions, and the cells obtained by $2 \mathrm{D}$ culture were collected following trypsin treatment. Subsequently, the spheroids and cells were used for treatments as described below.

Antibodies. Anti-Smad1 (cat. no. 6944), anti-Smad5 (cat. no. 12534), anti-phospho-Smad1 (cat. no. 5753), anti-phosphoSmad1/5 (cat. no. 9516), anti-phospho-Smad1/Smad5/Smad9 (cat. no. 13820) (all used at a 1:1,000 dilution; and all from Cell Signaling Technology, Danvers, MA, USA) and anti-SMAD4 (diluted 1:100; cat. no. sc-7966; Santa Cruz Biotechnology, Santa Cruz, CA, USA) antibodies were used for western blot analysis and immunocytochemistry as the primary antibodies. In addition, anti- $\beta$-actin antibody (diluted 1:5,000; cat. no. A5316; Sigma-Aldrich, St. Louis, MO, USA) was used as an internal control.

Morphological observation. Spheroids obtained by 3D culture were observed in culture using an inverted microscope (IX70; Olympus, Tokyo, Japan) and their diameters measured. The cultured cells were fixed with $10 \%$ neutral-buffered formalin. The sediment following centrifugation $(1,500 \mathrm{x} g$ and room temperature) was collected, and cell blocks were then prepared by the sodium alginate method. Normal paraffin-embedded sections were prepared from the cell blocks and used for hematoxylin and eosin (H\&E) staining and immunocytochemistry.

Immunocytochemical staining. Paraffin-embedded sections of obtained spheroids were prepared using the BenchMark GX Slide Staining System (Roche, Basel, Switzerland), and subjected to immunocytochemistry with each primary antibody following heat-induced epitope retrieval. A 3,3'-diamino benzidine reaction against peroxidase-labeled secondary antibody was used for visualization.

RNA extraction and cDNA preparation. The cultured cells were collected and rinsed with phosphate-buffered saline, and total RNA was then extracted from the cells using a PureLink RNA Mini kit (Ambion/Thermo Fisher Scientific) in accordance with the manufacturer's instructions. Subsequently, cDNA was prepared from the obtained total RNA using Transcriptor Universal cDNA Master (Roche) by a reverse transcription reaction in accordance with the manufacturer's instructions.

Quantitative PCR. Using the prepared cDNA mentioned above, quantitative PCR was implemented using FastStart Essential DNA Green Master (Roche) in accordance with the manufacturer's instructions. LightCycler Nano (Roche) was used for the reaction by setting denaturation at $95^{\circ} \mathrm{C}$ for $10 \mathrm{sec}$, annealing at $60^{\circ} \mathrm{C}$ for $10 \mathrm{sec}$, and extension at $72^{\circ} \mathrm{C}$ for $15 \mathrm{sec}$ per cycle, with the reaction conducted for 45 cycles. LightCycler Nano 
Table I. Primers used for quantitative PCR.

\begin{tabular}{|c|c|c|}
\hline $\begin{array}{l}\text { Gene } \\
\text { name }\end{array}$ & $\begin{array}{l}\text { Forward sequence } \\
\qquad\left(5^{\prime} \rightarrow 3^{\prime}\right)\end{array}$ & $\begin{array}{l}\text { Reverse sequence } \\
\qquad\left(5^{\prime} \rightarrow 3^{\prime}\right)\end{array}$ \\
\hline SMAD4 & cctgttcacaatgagcttgc & gcaatggaacaccaatactcag \\
\hline ENG & ttcctggagttcccaacg & aggtgccattttgcttgg \\
\hline BAMBI & cgccactccagctacatctt & cacagtagcatcgaatttcacc \\
\hline NOG & gaagctgcggaggaagttac & tacagcacggggcagaat \\
\hline TGFBR2 & gggaaatgacatctcgctgta & caccttggaaccaaatggag \\
\hline SMAD9 & tgagccagagagtccctatca & catagtaggcgaccgagcac \\
\hline$\beta$-actin & gttgctatccaggctgtgc & gcatcctgtcggcaatgc \\
\hline
\end{tabular}

SMAD4, SMAD family member 4; ENG, endoglin; BAMBI, BMP and activin membrane bound inhibitor; NOG, noggin; TGFBR2, transforming growth factor- $\beta$ receptor 2 ; SMAD9, SMAD family member 9.

Software (Roche) was used for the analysis. The relative mRNA expression levels were calculated for the expression levels of $\beta$-actin, which is a housekeeping gene, based on quantitative cycles $(\mathrm{Cq})$ using the relative $\mathrm{Cq}\left(2^{-\Delta \Delta \mathrm{Cq}}\right)$ method (20). The primers used in this study are listed in Table I.

Western blot analysis. Complete Lysis-M (Roche) was used for the extraction of protein from the cultured cells. Samples prepared at the same protein concentration were separated by sodium dodecyl sulfate-polyacrylamide gel electrophoresis and transferred onto polyvinylidene difluoride membranes. To prevent non-specific reactions, the membranes were blocked by $5 \%$ skim milk, and then treated with each primary antibody at $4^{\circ} \mathrm{C}$ overnight. The membranes were then reacted with HRP-labeled anti-rabbit (\#7074s) or anti-mouse (\#7076s) IgG (Cell Signaling Technology) as the secondary antibody. Protein expression was confirmed by chemiluminescence (Amersham, Little Chalfont, UK).

RNA interference. The KP-2 and KP-3 cells were preliminarily incubated for $24 \mathrm{~h}$ after adjusting the cell numbers. These cells were transfected with target siRNA (Nippon Gene, Toyama, Japan) using Opti-MEM (Gibco) and Lipofectamine ${ }^{\mathrm{TM}}$ RNAiMAX reagent (Invitrogen). The sequences of the siRNA used in this study were as follows: si-SMAD4 sense, 5'-GUA UGAUGGUGAAGGAUGAdTdT-3' and antisense, 5'-UCAUCC UUCACCAUCAUACdTdT-3'; si-ENG sense, 5'-CCAGCAUUG UCUCACUUCAdTdT-3' and antisense, 5'-UCAAGUGAGACA AUGCUGGdTdT-3'; and scrambled siRNA sense, 5'-CGUACG CGGAAUACUUCGAdTdT-3' and antisense, 5'-UCGAAGUAU UCCGCGUACGdTdT-3' (21,22). Following transfection, the cells were returned to RPMI-1640 medium, subjected to continued incubation for 18-24 h, and used in the following experiments.

Transwell cell invasion assay. A Corning BioCoat Matrigel Invasion Chamber (24-well plate, polycarbonate membrane, $8-\mu \mathrm{m}$ pore size) was used for the observation of morphological changes in spheroids associated with invasion and the measurement of the invasive capacity of individual tumor cells. Following the hydration of the Matrigel, a certain number of spheroids were inoculated into the upper chamber of each well. The morphology of the spheroids in the upper chamber was observed $0,3,6,12,18$ and $24 \mathrm{~h}$ following inoculation, and the number of spheroids was counted (BZ-X710; Keyence, Osaka, Japan). At $24 \mathrm{~h}$ following inoculation, the cells that had invaded to the lower side of a membrane were fixed with $10 \%$ neutral buffered formalin, stained with toluidine blue (Wako Pure Chemical Industries, Osaka, Japan), and then counted (BX50; Olympus). In addition, cells that had sunk in the lower chamber were observed.

Statistical analysis. The means \pm standard error were calculated for the measured mRNA expression levels and the diameters of the spheroids. A Student's t-test was used for a comparative test between groups. A $\mathrm{P}<0.05$ was considered to indicate a statistically significant difference.

\section{Results}

Spheroid formation by pancreatic cancer cell lines and their morphology. A total of 8 cell lines derived from human pancreatic cancer (AsPC-1, BxPC-3, KP-2, KP-3, MIA-PaCa, Panc-1, SUIT-2 and TCC-PAN2) grew as individual cells under ordinary $2 \mathrm{D}$ culture conditions. The KP-2 and KP-3 cells exhibited a mild tendency to form clumps, and only several cells bound together (Fig. 1A and B). By contrast, under 3D culture conditions, the KP-2 and KP-3 cells bound to each other and formed spherical clusters termed spheroids. The other cell lines exhibited weak binding between cells although they had a tendency to form clusters in culture medium; spheroid formation was infrequently observed (data not shown). Cell block specimens of spheroids obtained from KP-2 and KP-3 cells indicated these had a uniform inner structure; however, an obvious tissue structure with polarized configuration or glandular structure was rarely observed (Fig. 1C and D).

Expression of TGF- $\beta$ signaling factors. The cells lines exhibited differences in the expression level of SMAD4; almost none or very little, if any, expression was observed in the AsPC-1, BxPC-3, SUIT-2 and TCC-PAN2 cell lines. By contrast, the KP-2, Panc-1, KP-3 and MIA-PaCa cell lines exhibited significant SMAD4 expression, although differences in the degree of expression were evident (Fig. 2A). Furthermore, ENG, a component of the TGF- $\beta$ signaling system, was expressed in all cell lines in almost the same expression pattern as SMAD4; an elevated expression was observed in 3 cell lines (KP-2, Panc-1 and MIA-PaCa) (Fig. 2B). In a comparison between $2 \mathrm{D}$ and 3D culture conditions, however, a significant increase in SMAD4 and ENG expression was observed under the 3D conditions in a number of cell lines (Fig. 2). In an immunocytochemical analysis using cell block sections of KP-3 cells, SMAD4 was localized in both the nucleus and cytoplasm under 2D conditions; however, it was localized only in the nucleus under 3D conditions (Fig. 3).

Significant decreases in the mRNA and protein expression levels were observed following SMAD4 knockdown using siRNA in the KP-3 cells, in which SMAD4 was normally highly expressed (Fig. 4A). The mRNA expression levels of BMP and activin membrane-bound inhibitor (BAMBI) and noggin (NOG), which are also components of the TGF- $\beta$ 


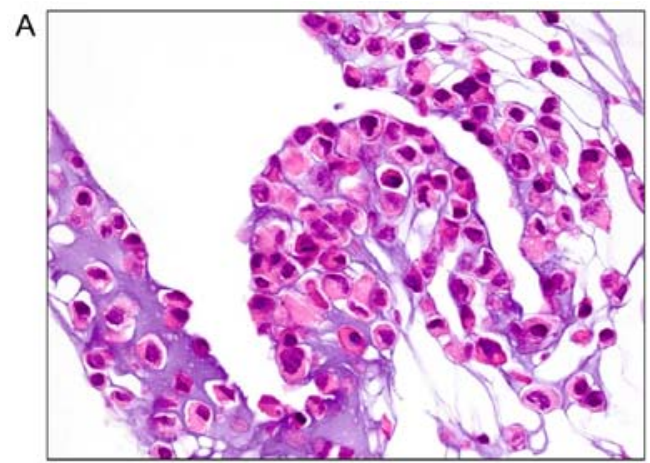

B
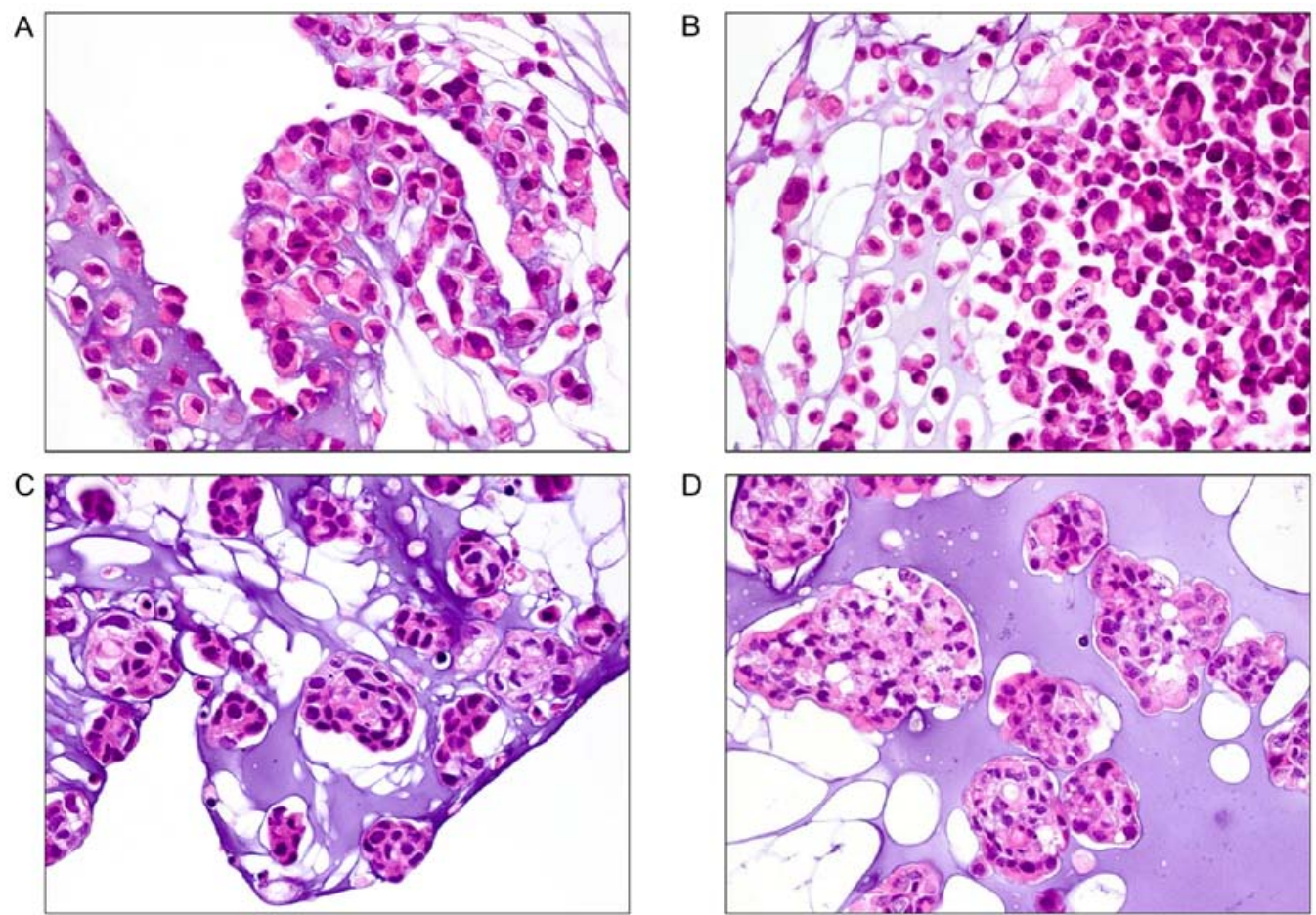

Figure 1. Morphological images of the KP-2 and KP-3 cells obtained under 2D and 3D culture conditions. (A and B) Under 2D culture conditions, the KP-2 (A) and KP-3 (B) cells exhibited a mild tendency to form clumps, and only several cells bound together. (C and D) By contrast, under 3D culture conditions, the KP-2 (C) and KP-3 (D) cells bound with each other and formed spheroids. The spheroids exhibited a uniform inner structure.
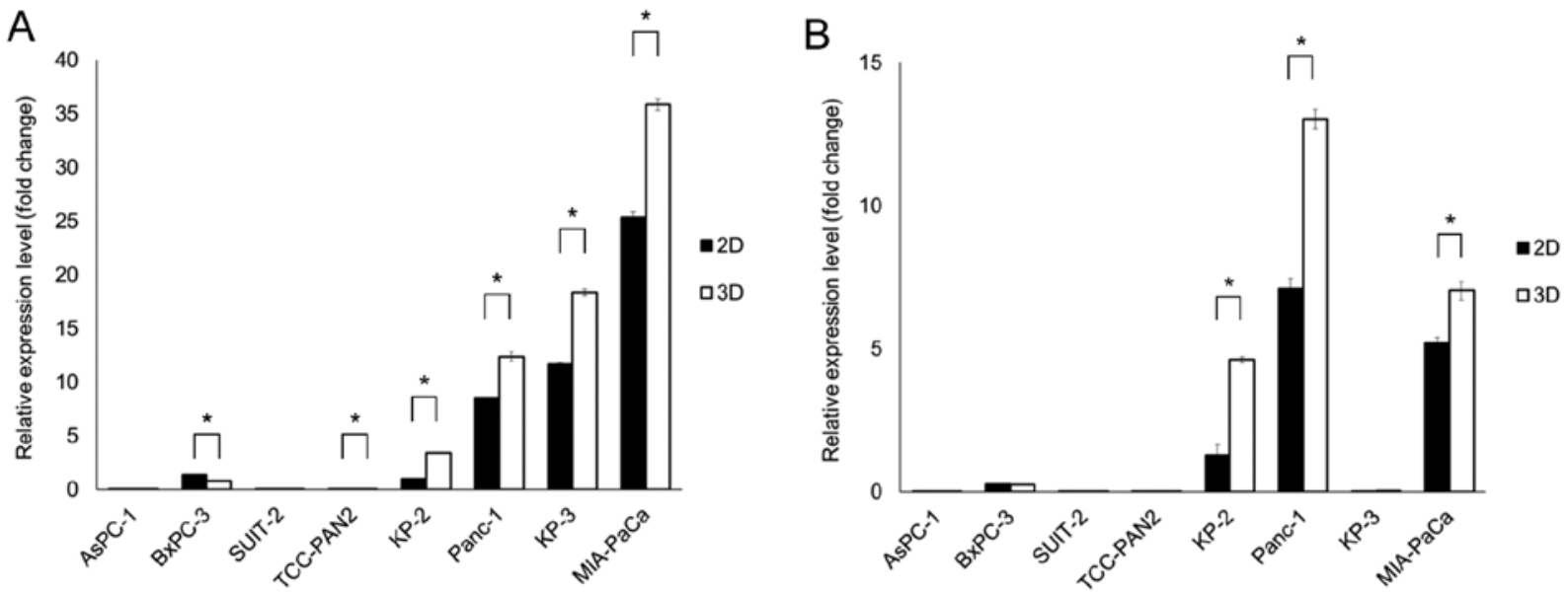

Figure 2. Relative mRNA expression levels of SMAD4 and endoglin (ENG) in various cell lines under 2D and 3D culture conditions. (A) The cell lines exhibited differences in the expression level of SMAD4; almost no or very little, if any, expression was observed in the AsPC-1, BxPC-3, SUIT-2 and TCC-PAN2 cell lines. By contrast, the KP-2, Panc-1, KP-3 and MIA-PaCa cell lines exhibited SMAD4 expression, although differences in the degree of expression were evident. (B) ENG was expressed in all cell lines in almost the same expression pattern as SMAD4; an elevated expression was observed in 3 cell lines (KP-2, Panc-1 and MIA-PaCa). (A and B) In a comparison between 2D and 3D culture conditions, however, significant increases in SMAD4 and ENG expression were observed under 3D conditions in many cell lines. Data shown are the means \pm SEM, $n=4 .{ }^{*} \mathrm{P}<0.05$.

signaling system, were increased in these cells (Fig. 4A). By contrast, no changes were observed in the mRNA expression of ENG in cells cultured under 2D conditions; however, its expression was decreased in the spheroid cells in the same manner as SMAD4 (Fig. 4A). However, the effects on the expression of phosphorylated SMAD1/5/9 and 2/3, which are SMAD4-binding proteins, were attenuated, particularly in the spheroid cells (Fig. 4B).

ENG knockdown using siRNA in the KP-2 cells led to the diameter of the spheroids becoming significantly smaller compared with the control group (Fig. 5A). With the suppression of ENG expression, a decrease in the mRNA expression levels of TGFBR2 and SMAD9 was observed in the spheroid cells (Fig. 5B). Furthermore, variations in phosphorylated SMAD1/5/9 protein expression were observed (Fig. 5C).

Transwell cell invasion assay. Morphological changes were observed in the spheroids over time following the inoculation of the upper chamber of Matrigel-coated wells in an invasion assay. The spheroids gradually become smaller, disrupted, 

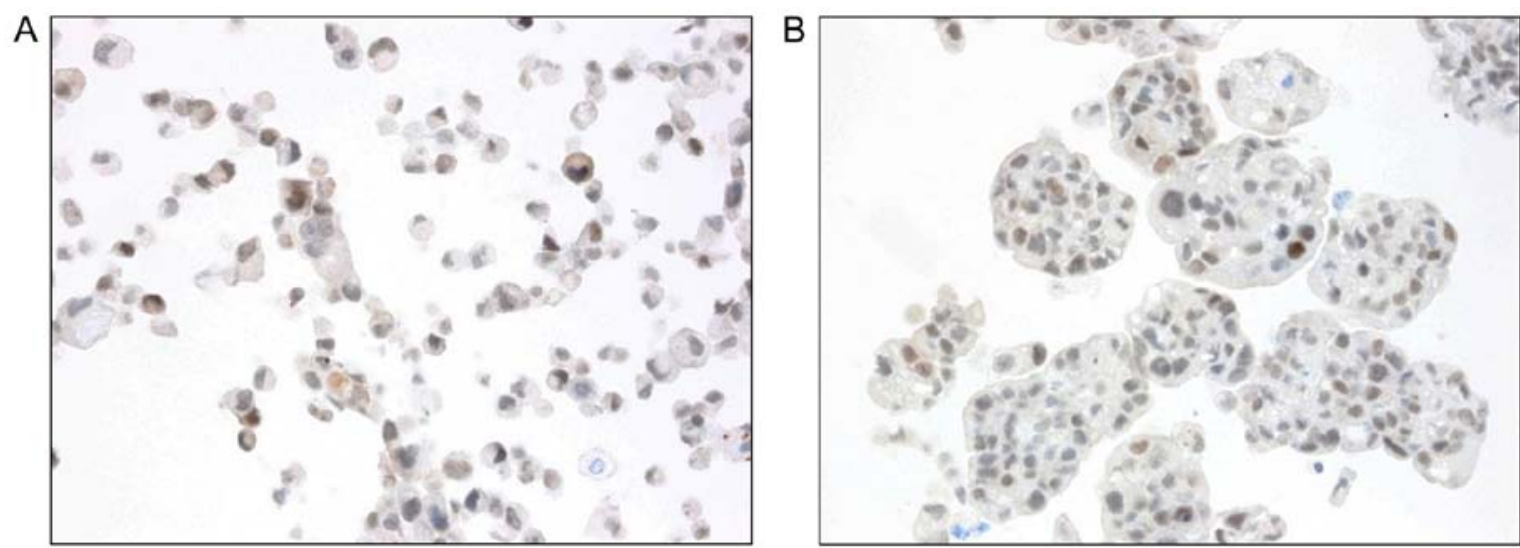

Figure 3. Immunocytochemical staining of SMAD4 in KP-3 cells. (A) SMAD4 localized in both the nucleus and cytoplasm under 2D conditions. (B) It localized only in the nucleus in most cells under $3 \mathrm{D}$ conditions.
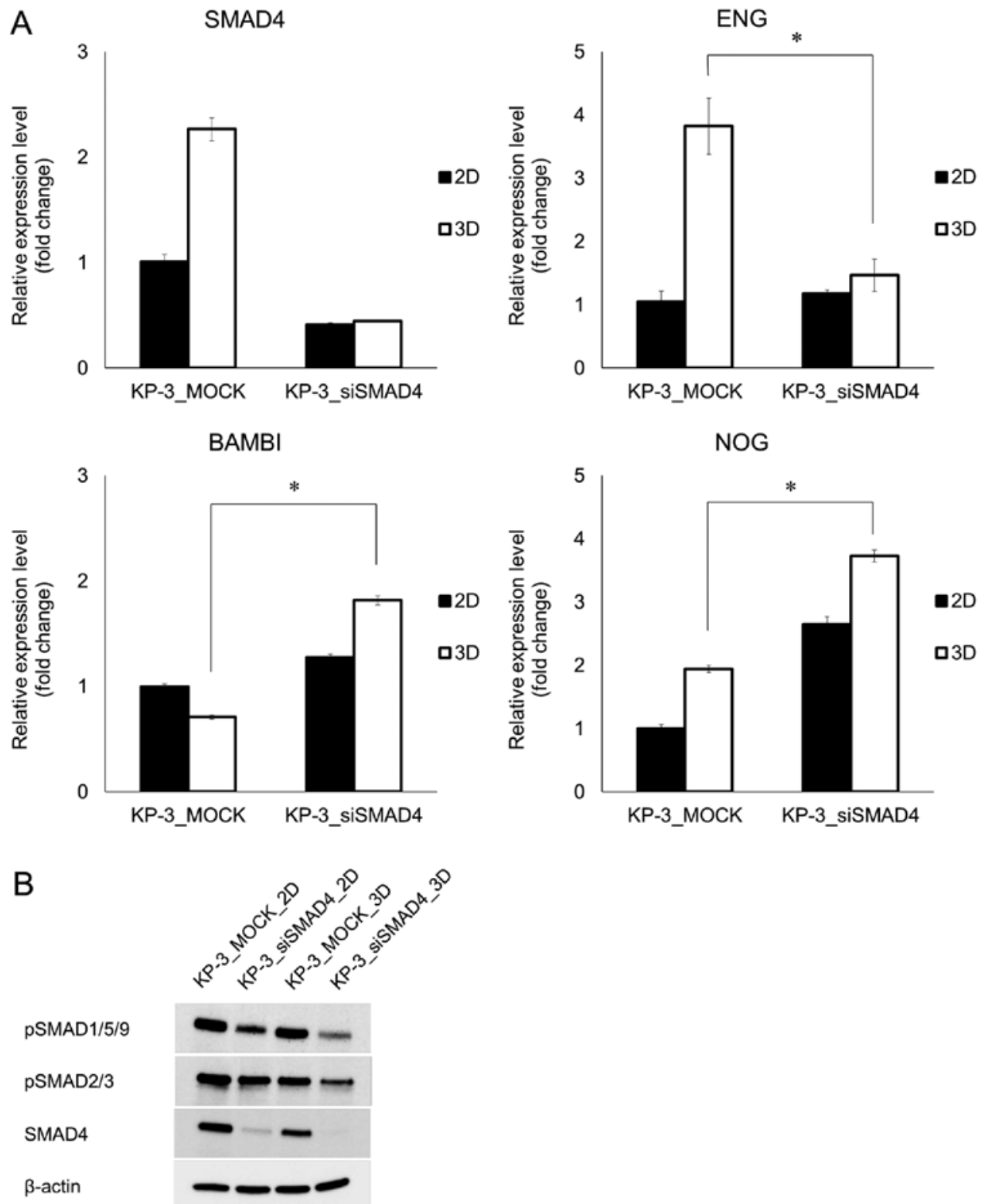

Figure 4. Effect on transforming growth factor- $\beta$ (TGF- $\beta$ ) signaling proteins after SMAD4 knockdown in KP-3 cells. (A) Significant decreases in the expression levels of mRNA were observed after SMAD4 knockdown by siRNA in the KP-3 cells. The mRNA expression of BMP and activin membrane-bound inhibitor (BAMBI) and noggin (NOG) was increased in these cells. By contrast, no changes were observed in the mRNA expression of endoglin (ENG) in 2D cultured cells; however it was attenuated in spheroid cells in the same manner as SMAD4. (B) Effects on the expression of phosphorylated SMAD1/5/9 and 2/3, which are SMAD4-binding proteins, were decreased, particularly in spheroid cells. Data shown are the means \pm SEM, $n=4$. "P<0.05. 

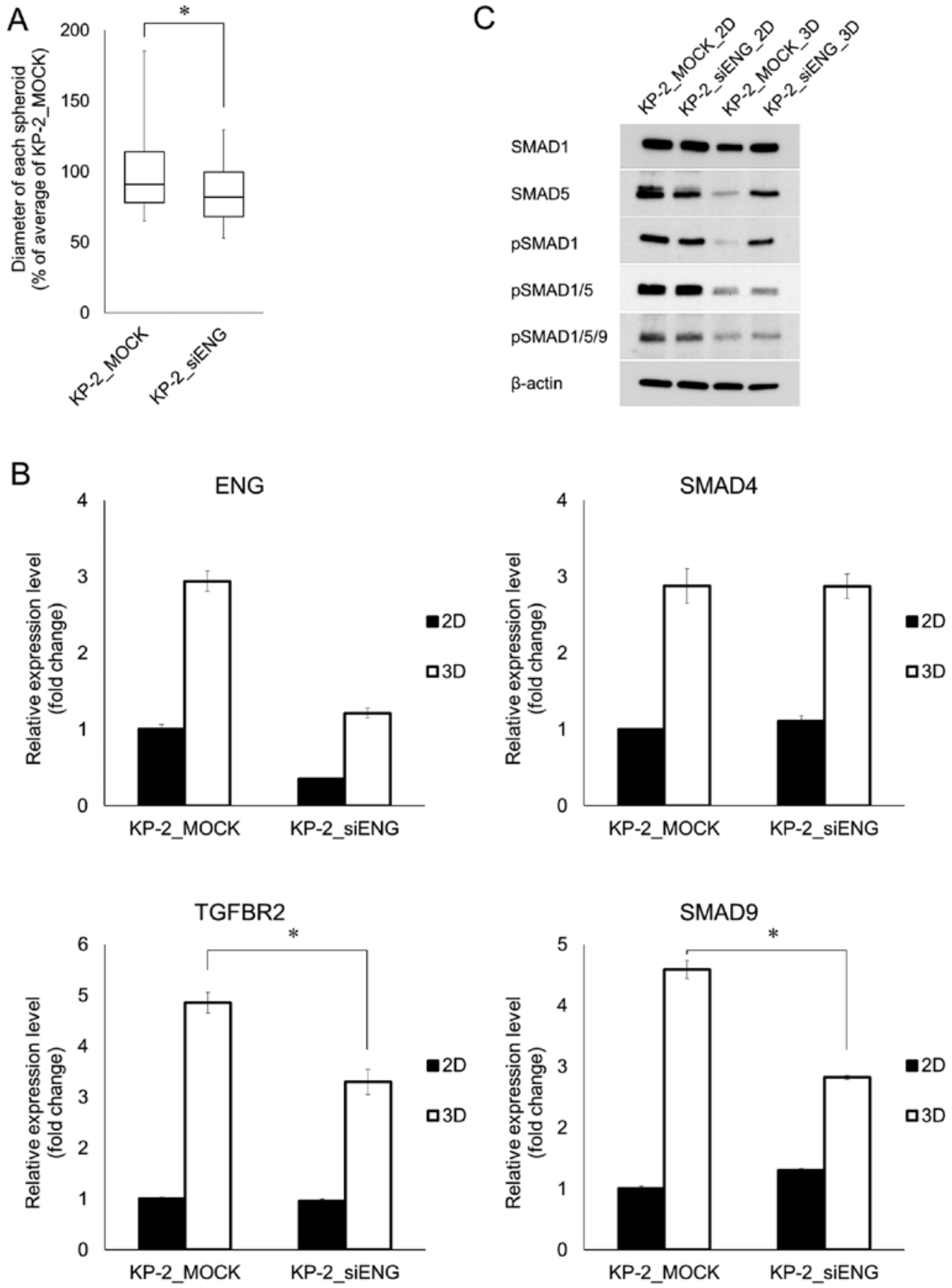

Figure 5. Effect on spheroid size and transforming growth factor- $\beta$ (TGF- $\beta$ ) signaling proteins after endoglin (ENG) knockdown in KP-2 cells. (A) ENG knockdown by siRNA in KP-2 cells led to the diameter of spheroids becoming significantly smaller compared with the control group. (B) With the suppression of ENG expression, a decrease in the mRNA expression levels of TGF- $\beta$ receptor type 2 (TGFBR2) and SMAD9 was observed in the spheroid cells. (C) Variations in phosphorylated SMAD1/5/9 protein expression were observed. Data shown are the means \pm SEM, $n=30$ (diameter), $n=4$ (quantitative PCR). ${ }^{*} \mathrm{P}<0.05$.

and solitary on Matrigel over time; the cells also invaded into the Matrigel. However, some spheroids remained, even $24 \mathrm{~h}$ following inoculation using untreated control cells (Fig. 6A). When a similar experiment was performed using cells in which ENG was knocked down, almost all spheroids became disrupted $12 \mathrm{~h}$ following inoculation (Fig. 6A), and the cells became extended and solitary (Fig. 6B). By $24 \mathrm{~h}$ following inoculation, the cells had invaded into the Matrigel insert, with fewer cells invading to the lower side of the membrane (for the cells in which ENG was knocked down) compared to the untreated group (Fig. 6C). Moreover, the number of cells that detached from the lower side of the membrane and became suspended in the lower chamber was quite large (for the ENG-knockdown cell group) (data not shown).

\section{Discussion}

Pancreatic cancer is associated with a very poor prognosis compared with malignant tumors in other organs. This poor prognosis is attributed to the specific characteristics of pancreatic cancer: it easily invades into adjacent organs, and, as a result, the frequency of remote metastasis is high (2). Pancreatic ductal carcinoma, which constitutes the majority of cases of pancreatic cancer, is characterized by a highly fibrotic 

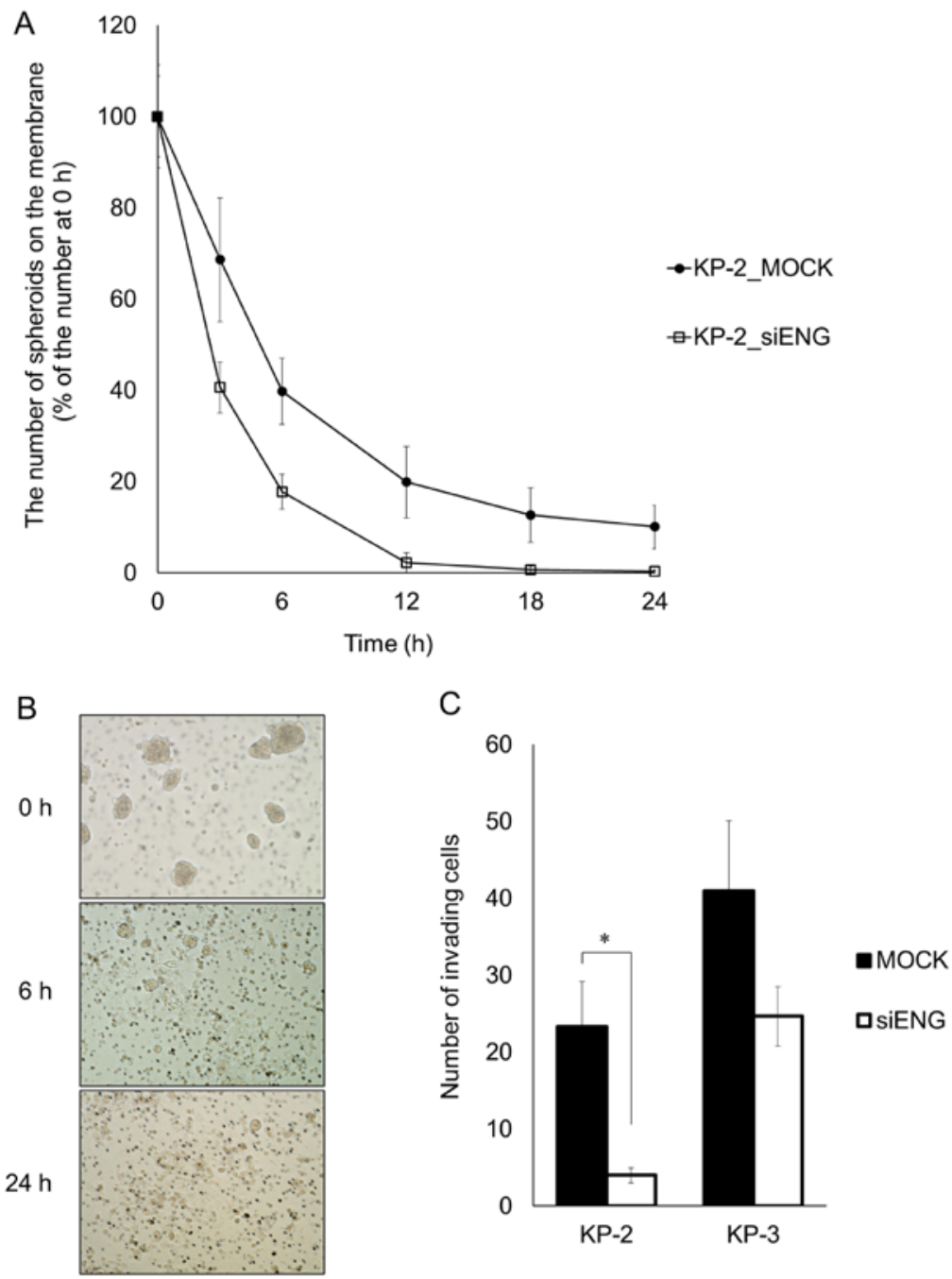

Figure 6. Transwell cell invasion assay with cells in which endoglin (ENG) was knocked down. (A and B) Spheroids gradually become smaller, disrupted, and solitary on Matrigel over time; cells also invaded into the Matrigel. However, some spheroids remained, even $24 \mathrm{~h}$ following inoculation using untreated control cells. When a similar experiment was performed using cells in which ENG was knocked down, almost all spheroids became disrupted $12 \mathrm{~h}$ following inoculation, and the cells became extended and solitary. (C) By $24 \mathrm{~h}$ following inoculation, the cells invaded into the Matrigel insert, with fewer cells invading to the lower side of the membrane for ENG-knockdown cells compared to the untreated control group. Data shown are the means \pm SEM, each performed in triplicate. ${ }^{*} \mathrm{P}<0.05$.

stroma; however, tumor cells exhibit a tendency to readily invade. Such tumor cells invade as single cells reflecting the EMT phenomenon; these cells are also characterized by the formation of an alveolar structure and invasion into fibrous stroma while maintaining a glandular cavity structure (4). These tendencies are not very often observed in malignant tumors in other organs. However, the mechanisms involved in pancreatic cancer cells invading into a distinctive fiber-rich stroma while maintaining a glandular cavity structure are still unclear. Accordingly, this study aimed to clarify the characteristic phenomena pancreatic cancer undergoes, particularly the mechanism of the invasion, while maintaining polarity and a glandular cavity structure, as well as identifying the factors involved.

An in vitro experimental system adequately reflecting in vivo conditions was used in this study. More specifically, a three-dimensional culture method that allows the formation of spheroids having a tissue structure similar to that under living conditions was used instead of a normal monolayer culture in which cells are grown adhering on the bottom of culture dish (23). As culture ware with a concave bottom surface with non-adherent treatment was used in this method, the cells formed three-dimensional spheroids by binding to each other. Not all pancreatic cell lines used in this study uniformly formed spheroids. The AsPC-1 and several cell lines formed small and loosely bound clusters, but strong spheroids were not formed (data not shown). By contrast, the KP-2 and KP-3 cell lines maintained their binding ability between cells and formed spherical spheroids; therefore, a difference in the ability to form spheroids was observed among the cell lines. This may be due to the various histological types of the cell lines. For example, the AsPC-1 cell line was originally derived from tumor tissue mainly consisting of poorly differentiated adenocarcinoma; however, the KP-2 and KP-3 cell lines were derived from differentiated adenocarcinomas. The former cell line invades as single cells; however, the latter exhibits a tendency to invade, forming an alveolar structure and/or maintaining a glandular structure. Such characteristics are related to the tendency of the latter two cell lines to easily form spheroids under 3D culture conditions. However, the 
cells did not show any arrangement with polarity, or directional or glandular formation in spheroids. We presume that this may have been, for example, as liver kinase B1, which is directly involved in cell polarity (24), or tight junction factors characterized by zonula occludens, claudin and other proteins act not only to maintain cell binding, but also to control cell polarity, thus inducing glandular formation within spheroids.

In order to elucidate the control mechanisms of spheroid formation and the factors involved in mechanisms, we compared and examined several signaling proteins expressed by the cells in 3D culture conditions with those expressed by the cells under normal 2D culture conditions. Several differences in the expression of various proteins between both groups were found. Focusing on the TGF- $\beta$ signaling system, we found the significantly enhanced expression of SMAD4 and ENG under 3D conditions, in particular. SMAD4 binds to R-SMAD phosphorylated by the stimulation of factors, such as TGF- $\beta$, BMP and activin, as well as others, and is then translocated into the nucleus to regulate the transcription of various proteins (13). In this study, the intracellular localization of SMAD4 was examined immunocytochemically. As a result, it was found to be localized in both the cytoplasm and nucleus when cells were grown under 2D culture conditions, whereas it localized only in the nucleus in cells cultured under 3D culture conditions. It was thus inferred that spheroid formation was promoted not only by the increased expression of SMAD4 in cells, but also by the enhancement of transcription of other proteins in the nucleus caused by the translocation of SMAD4 to the nucleus from the cytoplasm in the process of forming spheroids.

In comparison, ENG is known as an accessory receptor of TGF- $\beta$ that localizes on the cell membrane, is expressed by vascular endothelial cells at a high level and is involved in angiogenesis (12). In the cell lines used in this study, ENG highlighted cellular behaviors similar to those of SMAD4. In other words, ENG and SMAD4 were expressed in the same manner in cell lines that easily formed spheroids, and the expression of these factors was increased when spheroids were formed. This suggests that both ENG and SMAD4 are involved in spheroid formation. However, whether these proteins function independently or cooperatively remains unknown. In this study, SMAD4 knockdown by siRNA was performed in the KP-3 cell line, which easily forms spheroids and expresses SMAD4 at a high level. As a result, an increase in the expression levels was observed for NOG and BAMBI, and a decrease in expression level was observed for ENG. These results suggest that SMAD4 may control the expression of ENG. In addition, NOG and BAMBI are believed to be inhibitory factors of the TGF- $\beta$ signaling pathway, while ENG is an enhancement factor of the pathway $(25,26)$. These findings suggest that SMAD4 may control the expression of ENG by acting in a positive feedback manner in the TGF- $\beta$ pathway.

Furthermore, in the same manner, variations in the phosphorylation of pSMAD1/5/9 were observed when ENG was knocked down in the KP-2 cells. In addition, spheroids derived from these cells in which ENG was knocked down exhibited tendencies to form increased smaller-sized spheroids on Matrigel and spheroids prone to disruption. These observations may explain a certain aspect of the EMT phenomenon in spheroids, such as a decrease in the binding ability of cells and an ability to be disrupted easily. However, fewer cells adhered on the lower side of the membrane in cells in which ENG was knocked down. This result may initially indicate that the invasive capacity was inhibited by ENG knockdown. However, as a number of cells existed in the lower chamber, this may indicate that the invasive capacity was actually enhanced and that adhesiveness was also reduced in the cells in which ENG was knocked down. Furthermore, it is also known that the extracellular portion of ENG is removed by matrix metalloprotease-14 and released extracellularly as soluble ENG (27). In addition, the blood ENG level is increased for some malignant tumors (28). ENG present in the cell membrane may inhibit the development of a tumor, that is, its invasiveness, by promoting the mutual binding of cells. However, tumor cells may lose their connectivity and acquire an invasive ability by the breakage and release of ENG. We consider that if a moleculartargeted therapeutic agent that prevents the cellular release of ENG is developed, it may lead to a means of suppressing the invasiveness of pancreatic cancer.

In conclusion, this study suggests that ENG may be regulated by a pathway mediated by SMAD4, an observation that has not attracted attention thus far in studies on pancreatic cancer. In addition, ENG was involved in the maintenance of binding among tumor cells and the inhibition of invasion. Therefore, it may be possible to specifically suppress invasiveness, which determines the prognosis of pancreatic cancer, by elucidating the mechanisms of ENG expression. This may be a promising field of research in future.

\section{Acknowledgements}

The authors would like to thank Dr M. Sasahara, Dr Y. Ishii, Dr T. Hamashima, Dr S. Yamamoto (Department of Pathology, Graduate School of Medicine and Pharmaceutical Sciences for Research,University of Toyama, Toyama,Japan),DrK. Tsuneyama (Department of Pathology and Laboratory Medicine, Tokushima University Graduate School, Tokushima, Japan) and Dr S. Hayashi (Department of Diagnostic Pathology, Takaoka City Hospital, Toyama, Japan) for their helpful advice and support of this study. The authors would also like to thank Mr. K. Hamada (Department of Diagnostic Pathology, Toyama University Hospital, Toyama, Japan) for his technical assistance.

\section{Funding}

No funding was received.

\section{Availability of data and materials}

The analyzed data sets generated during the study are available from the corresponding author on reasonable request.

\section{Authors' contributions}

EK designed the study, and wrote the initial draft of the manuscript. JI contributed to analysis and interpretation of data, and assisted in the preparation of the manuscript. AS, TM, TNa, SM, HH, TNi and CK contributed to data collection and interpretation, and critically reviewed the manuscript. All authors have read and approved the final manuscript. 


\section{Ethics approval and consent to participate}

Not applicable.

\section{Consent for publication}

Not applicable.

\section{Competing interests}

The authors declare that they have no competing interests.

\section{References}

1. Siegel RL, Miller KD and Jemal A: Cancer Statistics, 2017. CA Cancer J Clin 67: 7-30, 2017.

2. Stathis A and Moore MJ: Advanced pancreatic carcinoma: Current treatment and future challenges. Nat Rev Clin Oncol 7 $163-172,2010$.

3. Hruban RH, Pitman MB and Klimstra DS: Tumors of the Pancreas. In: Atlas of Tumor Pathology. 4th series edition. Vol 6. Washington DC, 2007.

4. HrubanRH,TakaoriK,Klimstra DS, Adsay NV,Albores-SaavedraJ, Biankin AV, Biankin SA, Compton C, Fukushima N, Furukawa T, et al: An illustrated consensus on the classification of pancreatic intraepithelial neoplasia and intraductal papillary mucinous neoplasms. Am J Surg Pathol 28: 977-987, 2004.

5. Jones S, Zhang X, Parsons DW, Lin JC, Leary RJ, Angenendt P, Mankoo P, Carter H, Kamiyama H, Jimeno A, et al: Core signaling pathways in human pancreatic cancers revealed by global genomic analyses. Science 321: 1801-1806, 2008.

6. Waddell N, Pajic M, Patch AM, Chang DK, Kassahn KS, Bailey P, Johns AL, Miller D, Nones K, Quek K, et al; Australian Pancreatic Cancer Genome Initiative: Whole genomes redefine the mutational landscape of pancreatic cancer. Nature 518: 495-501, 2015.

7. Almoguera C, Shibata D, Forrester K, Martin J, Arnheim N and Perucho M: Most human carcinomas of the exocrine pancreas contain mutant c-K-ras genes. Cell 53: 549-554, 1988.

8. Caldas C, Hahn SA, da Costa LT, Redston MS, Schutte M, Seymour AB, Weinstein CL, Hruban RH, Yeo CJ and Kern SE: Frequent somatic mutations and homozygous deletions of the p16 (MTS1) gene in pancreatic adenocarcinoma. Nat Genet 8: 27-32, 1994.

9. Redston MS, Caldas C, Seymour AB, Hruban RH, da Costa L, Yeo CJ and Kern SE: p53 mutations in pancreatic carcinoma and evidence of common involvement of homocopolymer tracts in DNA microdeletions. Cancer Res 54: 3025-3033, 1994.

10. Hahn SA, Schutte M, Hoque AT, Moskaluk CA, da Costa LT, Rozenblum E, Weinstein CL, Fischer A, Yeo CJ, Hruban RH, et al: DPC4, a candidate tumor suppressor gene at human chromosome 18q21.1. Science 271: 350-353, 1996.

11. Lagna G, Hata A, Hemmati-Brivanlou A and Massagué J: Partnership between DPC4 and SMAD proteins in TGF-beta signalling pathways. Nature 383: 832-836, 1996.

12. Blackford A, Serrano OK, Wolfgang CL, Parmigiani G, Jones S, Zhang X, Parsons DW, Lin JC, Leary RJ, Eshleman JR, et al: SMAD4 gene mutations are associated with poor prognosis in pancreatic cancer. Clin Cancer Res 15: 4674-4679, 2009.
13. Massagué J: TGFbeta in Cancer. Cell 134: 215-230, 2008.

14. Nassiri F, Cusimano MD, Scheithauer BW, Rotondo F, Fazio A, Yousef GM, Syro LV, Kovacs K and Lloyd RV: Endoglin (CD105): A review of its role in angiogenesis and tumor diagnosis, progression and therapy. Anticancer Res 31: 2283-2290, 2011.

15. Gromova P, Rubin BP, Thys A, Cullus P, Erneux C and Vanderwinden JM: ENDOGLIN/CD105 is expressed in KIT positive cells in the gut and in gastrointestinal stromal tumours. J Cell Mol Med 16: 306-317, 2012.

16. Davidson B, Stavnes HT, Førsund M, Berner A and Staff AC: CD105 (Endoglin) expression in breast carcinoma effusions is a marker of poor survival. Breast 19: 493-498, 2010.

17. Ziebarth AJ, Nowsheen S, Steg AD, Shah MM, Katre AA, Dobbin ZC, Han HD, Lopez-Berestein G, Sood AK, Conner M, et al: Endoglin (CD105) contributes to platinum resistance and is a target for tumor-specific therapy in epithelial ovarian cancer. Clin Cancer Res 19: 170-182, 2013.

18. Fujiwara K, Ohuchida K, Ohtsuka T, Mizumoto K, Shindo K, Ikenaga N, Cui L, Takahata S, Aishima S and Tanaka M: Migratory activity of $\mathrm{CD} 105^{+}$pancreatic cancer cells is strongly enhanced by pancreatic stellate cells. Pancreas 42: 1283-1290, 2013.

19. Pal K, Pletnev AA, Dutta SK, Wang E, Zhao R, Baral A, Yadav VK, Aggarwal S, Krishnaswamy S, Alkharfy KM, et al: Inhibition of endoglin-GIPC interaction inhibits pancreatic cancer cell growth. Mol Cancer Ther 13: 2264-2275, 2014.

20. Livak KJ and Schmittgen TD: Analysis of relative gene expression data using real-time quantitative PCR and the 2(-Delta Delta C(T)) method. Methods 25: 402-408, 2001.

21. Ke Z,Zhang X, Ma L and Wang L: Deleted in pancreatic carcinoma locus $4 / \mathrm{Smad} 4$ participates in the regulation of apoptosis by affecting the Bcl-2/Bax balance in non-small cell lung cancer. Hum Pathol 39: 1438-1445, 2008.

22. Xu Y, Wang D, Zhao LM, Zhao XL, Shen JJ, Xie Y, Cao LL, Chen ZB, Luo YM, Bao BH, et al: Endoglin is necessary for angiogenesis in human ovarian carcinoma-derived primary endothelial cells. Cancer Biol Ther 14: 937-948, 2013.

23. Ishiguro T, Ohata H, Sato A, Yamawaki K, Enomoto T and Okamoto K: Tumor-derived spheroids: Relevance to cancer stem cells and clinical applications. Cancer Sci 108: 283-289, 2017.

24. Forcet $C$ and Billaud M: Dialogue between LKB1 and AMPK: A hot topic at the cellular pole. Sci STKE 2007: pe51, 2007.

25. Groppe J, Greenwald J, WiaterE,Rodriguez-LeonJ,Economides AN, Kwiatkowski W, Affolter M, Vale WW, Izpisua Belmonte JC and Choe S: Structural basis of BMP signalling inhibition by the cystine knot protein Noggin. Nature 420: 636-642, 2002.

26. Onichtchouk D, Chen YG, Dosch R, Gawantka V, Delius H, Massagué J and Niehrs C: Silencing of TGF-beta signalling by the pseudoreceptor BAMBI. Nature 401: 480-485, 1999.

27. Hawinkels LJ, Kuiper P, Wiercinska E, Verspaget HW, Liu Z, Pardali E, Sier CF and ten Dijke P: Matrix metalloproteinase-14 (MT1-MMP)-mediated endoglin shedding inhibits tumor angiogenesis. Cancer Res 70: 4141-4150, 2010.

28. Takahashi N, Kawanishi-Tabata R, Haba A, Tabata M, Haruta Y, Tsai $\mathrm{H}$ and Seon BK: Association of serum endoglin with metastasis in patients with colorectal, breast, and other solid tumors, and suppressive effect of chemotherapy on the serum endoglin. Clin Cancer Res 7: 524-532, 2001. 